\title{
Spin-Dependent Extended X-Ray-Absorption Fine Structure: Probing Magnetic Short-Range Order
}

\author{
G. Schütz, ${ }^{(1)}$ R. Frahm, (2) P. Mautner, ${ }^{(1)}$ R. Wienke, ${ }^{(1)}$ W. Wagner, ${ }^{(1)}$ W. Wilhelm, ${ }^{(1)}$ and P. Kienle ${ }^{(1)}$ \\ (1) Physik Department, Technische Universität München, D-8046 Garching, West Germany \\ (2) Hamburger Synchrotronstrahlungslabor (HASYLAB) am Deutschen Elektronen-Synchrotron (DESY),
} D-2000 Hamburg 52, West Germany

(Received 23 February 1989)

\begin{abstract}
The first evaluation of spin-dependent extended x-ray-absorption fine structure (SPEXAFS) is reported. The SPEXAFS at the Gd $L$ edges for $\mathrm{Gd}$ metal and $\mathrm{Gd}_{3} \mathrm{Fe}_{5} \mathrm{O}_{12}$ are compared to the conventional EXAFS and discussed in terms of exchange scattering from magnetic neighbors. The SPEXAFS at the $\mathrm{Gd} L_{2}$ and $L_{3}$ edges reflect the change of the photoelectron spin polarization. The Fourier transform of the SPEXAFS of the $\mathrm{Gd}_{3} \mathrm{Fe}_{5} \mathrm{O}_{12}$ only shows the magnetic neighborhood, whereas the nonmagnetic oxygen neighbors remain invisible. SPEXAFS spectroscopy is expected to become a powerful tool in the determination of the magnetic short-range order.
\end{abstract}

PACS numbers: $61.10 . \mathrm{Lx}, 75.25 .+\mathrm{z}, 75.50 .-\mathrm{y}, 78.70 . \mathrm{Dm}$

The measurement of the extended $x$-ray-absorption fine structure (EXAFS) with unpolarized or linearly polarized $\mathrm{x}$ rays is an established method to investigate the short-range order in a variety of materials. The EXAFS is the modulation of the photoabsorption cross section due to the interference of the de Broglie wave of the ejected photoelectron with the one which is scattered by the surrounding atoms. The EXAFS is calculated starting approximately at $30 \mathrm{eV}$ above an absorption edge by $\chi(k)=\Delta \mu / \mu_{0}$, where $k$ is the wave vector of the ejected photoelectron, $\Delta \mu$ is the oscillatory part of the absorption coefficient, and $\mu_{0}$ is a structureless background. In the interpretation and analysis of the EXAFS measurements with unpolarized or linearly polarized $\mathrm{x}$ rays only the Coulomb interaction is considered for the elastic and inelastic scattering processes and thus the backscattering amplitude. However, we demonstrate that an additional exchange potential is present in magnetized materials.

It has been shown recently that the near-edge absorption of circularly polarized $\mathrm{x}$ rays in magnetized targets depends on the spin polarization of the photons relative to the spin polarization of the magnetic electrons in the absorber. ${ }^{1-3}$ This effect is interpreted in terms of the transfer of a spin-polarized photoelectron from an unpolarized initial core state into spin-split empty states near the Fermi level. The spin-polarized photoelectrons are created by a transfer of the photon polarization to the photoelectron in the direction of the $k$ vector of the photons due to the influence of spin-orbit interaction on the dipole matrix element. ${ }^{4}$ The photoelectron polarization $P_{e}$ can be calculated for free atoms. For photoelectron energies $E_{e} \ll m_{e} c^{2}$ the value of $P_{e}$ is nearly energy independent in the dipole approximation. Thus after absorption of circularly polarized $\mathrm{x}$ rays the outgoing photoelectrons are spin polarized not only in the near-edge region, but also in the EXAFS energy range. If the absorber is magnetized in the direction of the $k$ vector of the circularly polarized photons the electrons of the mag- netic neighboring atoms are also partially spin polarized and an exchange potential is present in addition to the Coulomb potential in the scattering process. This effect should also be observable in the EXAFS region.

An analogous phenomenon is observed for the diffraction of spin-polarized low-energy electrons (SPLEED) $)^{5-7}$ by ferromagnetic samples, where an asymmetry of the scattered intensity is found for parallel and antiparallel spin polarization of scattered electrons and magnetic electrons in the targets. This effect is attributed to a spin-dependent part of the scattering amplitude due to exchange scattering, which is about 2 orders of magnitude smaller than the Coulomb scattering amplitude.

The spin-dependent part of the backscattering amplitude results in different EXAFS in the case of photon spin parallel $\left(\chi^{+}\right)$and antiparallel $\left(\chi^{-}\right)$to the spin of the magnetic electrons in the absorber. The difference in the spin-polarized EXAFS (SPEXAFS) can be determined by measuring the spin-dependent absorption in the EXAFS energy range. The spin-dependent absorption coefficient is given by the normalized difference of the absorption coefficient $\mu_{c} / \mu=\left(\mu^{+}-\mu^{-}\right) /\left(\mu^{+}+\mu^{-}\right)$ for photons with spin parallel $\left(\mu^{+}\right)$and antiparallel $\left(\mu^{-}\right)$to the spin of the magnetic electrons in the absorber. The values $\mu^{ \pm}$represent the $L$-edge absorption after subtraction of a Victoreen-type background. ${ }^{8}$ If the "conventional" EXAFS oscillations $\chi=\left(\chi^{+}+\chi^{-}\right) / 2$ are not too large the spin-dependent EXAFS is directly related to the spin-dependent absorption coefficient by $\mu_{c} / \mu \approx\left(\chi^{+}-\chi^{-}\right) / 2$.

The SPEXAFS experiments were performed at the synchrotron radiation facility HASYLAB in Hamburg. The spectra were taken during runs of the storage ring DORIS II at an electron energy of $3.7 \mathrm{GeV}$ and an injection current of approximately $90 \mathrm{~mA}$. Two photon beams with opposite sense of circular polarization of $\left|P_{c}\right| \sim 0.8$ emitted from a bending magnet above and 

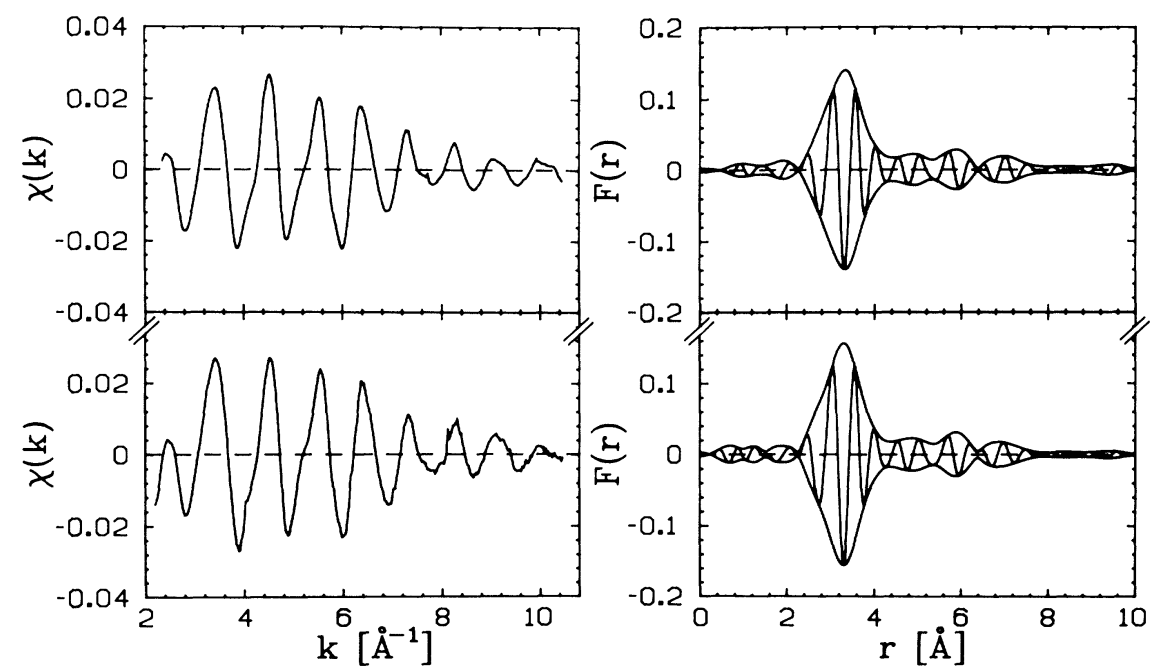

FIG. 1. EXAFS of Gd metal measured at $100 \mathrm{~K}$ at the $L_{3}$ edge (top) and the $L_{2}$ edge (bottom) and Fourier transforms (modulus and imaginary part) of both spectra, calculated with a Kaiser-Bessel window function over the spectral range $k^{2}=2.3-10.4 \AA^{-1}$ with a $k^{1}$ weighting.

below the orbit plane of the electrons in the storage ring were used. The double-crystal monochromator ROMO II was equipped with $\mathrm{Si}$ (311) crystals, which provided an energy resolution of $\sim 1 \mathrm{eV}$ in the energy range of 7200 to $8300 \mathrm{eV}$. The spin-dependent absorption coefficient was measured in transmission simultaneously for both photon beams with double-ionization chambers. The samples, a pure $\mathrm{Gd}$ metal foil and $\mathrm{Gd}_{3} \mathrm{Fe}_{5} \mathrm{O}_{12}$ powder with an effective thickness of approximately 4 $\mathrm{mg} / \mathrm{cm}^{2}$, were cooled to $100 \mathrm{~K}$ during the measurements. The external magnetic field of $\sim 80 \mathrm{mT}$ was reversed every second. The total data collection time was $5 \mathrm{~h}$ for the $\mathrm{Gd} L_{2}$-edge spectrum in $\mathrm{Gd}$ metal and $2 \mathrm{~h}$ for
$\mathrm{Gd}_{3} \mathrm{Fe}_{5} \mathrm{O}_{12}$. The $\mathrm{Gd} L_{3}$-edge spectrum was recorded in about $3 \mathrm{~h}$. A detailed description of the experimental method was given in Ref. 3.

The EXAFS data for the $L_{2}$ and $L_{3}$ edges in Gd metal are shown in Fig. 1. Details of the data reduction procedure are described in Ref. 8. All EXAFS data are displayed up to $k=10.4 \AA^{-1}$ which is the limit at the $L_{2}$ edge due to the onset of the $L_{1}$ absorption. As expected from standard EXAFS theory, both $\chi(k)$ spectra are in good agreement with respect to their periodicity, which is also reflected in the position of the nearest-neighbor peaks in the Fourier transforms at $3.32 \AA$. However, the peak positions have to be corrected by typically $0.3-0.4$
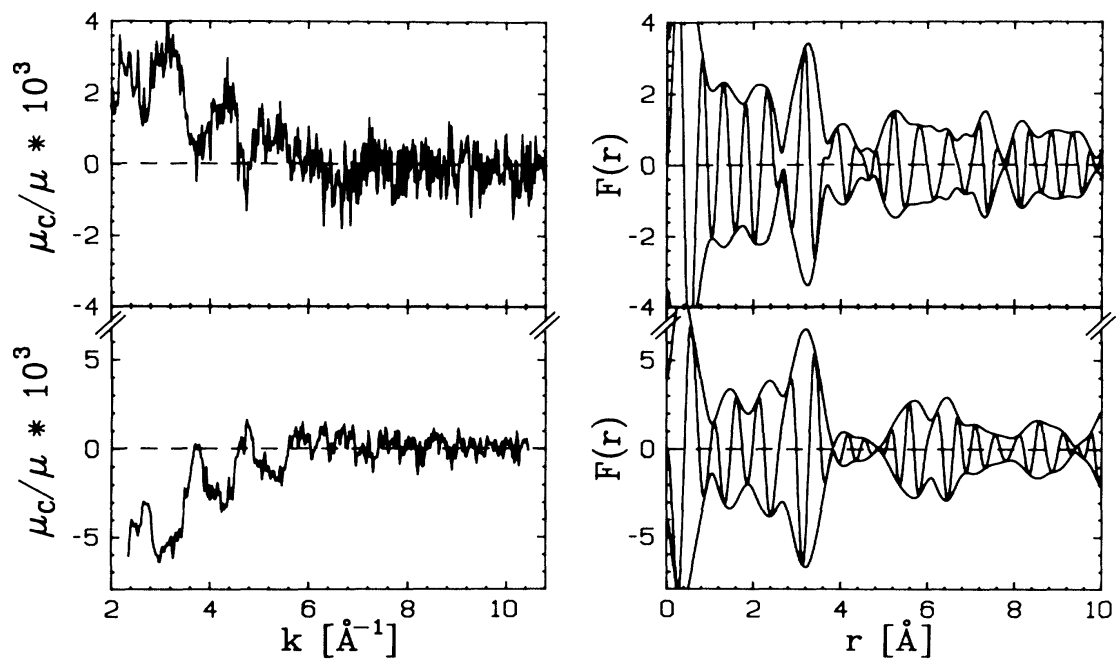

FIG. 2. SPEXAFS of Gd metal measured at $100 \mathrm{~K}$ at the $L_{3}$ edge (top) and the $L_{2}$ edge (bottom) and Fourier transforms (modulus and imaginary part) of both spectra, calculated with a Kaiser-Bessel function over the spectral range $k=2.3-10.4 \AA^{-1}$ with a $k^{1}$ weighting. 
$\AA$ for the influence of the scattering phase to get the real atomic distances. In the case of the hcp Gd metal the nearest-neighbor environment consists of two unresolvable shells with six atoms each at 3.573 and $3.636 \AA$. Because of the relatively low Debye temperature of $\mathrm{Gd}$ $(200 \mathrm{~K})$ the EXAFS structure is weak. Therefore the sample must be cooled down further for investigation of atomic shells at larger distances. The peak heights of the Fourier transforms shown in Fig. 1 are slightly different. This can be due to the overlap of the $L_{2}$ with the $L_{3}$ edge because the $L_{2}$-EXAFS data are influenced by the decaying signal of the $L_{3}$ EXAFS. This overlap may distort the EXAFS at the $L_{3}$ edge and also modify its Fourier transform as observed in the case of metallic gold. ${ }^{9}$

The spin-dependent EXAFS data for the $L_{2}$ and $L_{3}$ edges in Gd metal are displayed in Fig. 2. The spindependent EXAFS $\left[\mu_{c} / \mu\right](k)$ at the Gd-metal $L_{3}$ edge shows oscillations in addition to a positive smooth background which is decreasing with increasing $k$ values. The SPEXAFS has a maximum amplitude of about $\Delta\left(\mu_{c} / \mu\right) \sim 1.5 \times 10^{-3}$. Their frequency coincides with the oscillations in the corresponding $\chi(k)$ spectra (see Fig. 1). At the $\mathrm{Gd} L_{2}$ edge in $\mathrm{Gd}$ metal the sign of the $\left[\mu_{c} / \mu\right](k)$ profile is reversed. Oscillations with maximum amplitudes of $\Delta\left(\mu_{c} / \mu\right)_{\max } \sim 3 \times 10^{-3}$ are superimposed on a negative background, which is about twice as large as in the Gd $L_{3}$ spectrum. The phase of the periodic structure at the $L_{2}$ edge seems to be shifted roughly by $\pi$ with respect to the oscillations in the spinindependent EXAFS $\chi(k)$. In the Fourier transforms of the SPEXAFS one significant nearest-neighbor peak is visible at about $r=3.20 \AA$. This value is nearly the same as in the case of the spin-independent EXAFS. In the

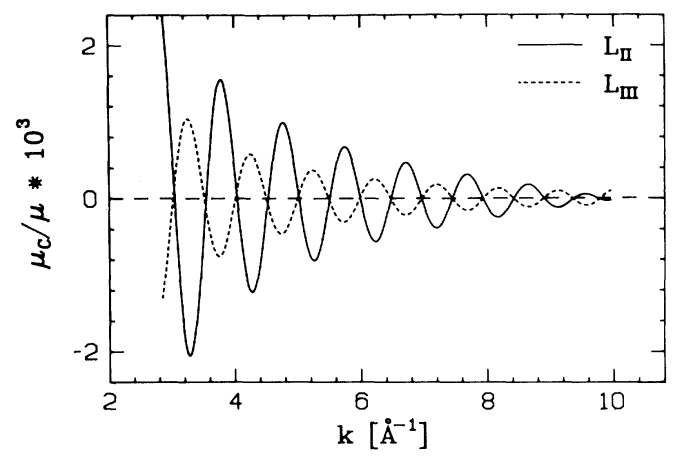

FIG. 3. Backtransforms of the nearest-neighbor peaks of the Gd SPEXAFS shown in Fig. 2. Full line: $L_{2}$ edge; dashed line: $L_{3}$ edge.

case of the Fourier transform of the SPEXAFS a large structure is visible at small $r$ values, which is due to the background discussed above and is of no relevance for the discussion of the nearest-neighbor peak. By inspection of the imaginary parts of the nearest-neighbor peaks it is obvious that the SPEXAFS oscillations change sign. This is verified by the backtransforms shown in Fig. 3. Besides this change of sign of the $L_{2}$ and $L_{3}$ spectra, also a difference in amplitude of a factor of $\sim-2$ is observable.

In the theoretical expression for the EXAFS the electron-electron interaction in the scattering process is described by the Coulomb scattering amplitude $F$, the phase $\Phi$, and the electron mean free path $\lambda$. Because of the influence of the exchange interaction these values will in principle contain additive spin-dependent parts $F_{c}$, $\Phi_{c}$, and $\lambda_{c}$ in the case of a spin polarization of the outgo-
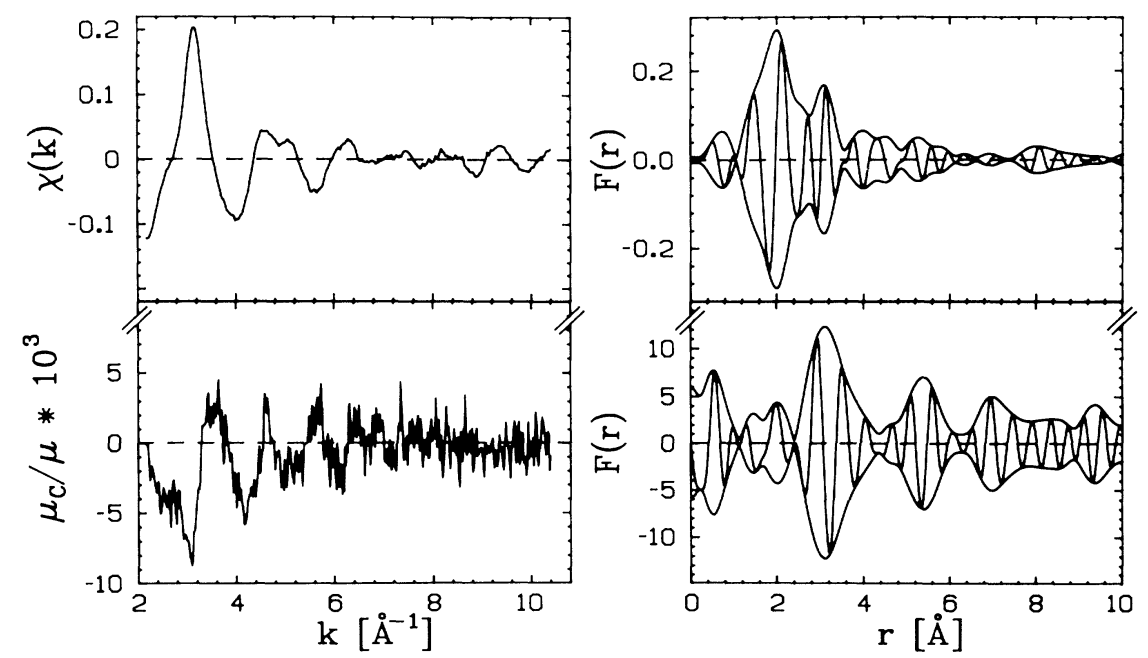

FIG. 4. EXAFS (top) and SPEXAFS (bottom) spectra of $\mathrm{Gd}_{3} \mathrm{Fe}_{5} \mathrm{O}_{12}$ measured at $100 \mathrm{~K}$ at the $L_{2}$ edge and Fourier transforms (modulus and imaginary part) of both spectra, calculated with a Kaiser-Bessel window function over the spectral range $k=2.3-10.4$ $\AA^{-1}$ with a $k^{1}$ weighting. 
ing electrons and scattering neighboring atoms, whose signs depend on the relative spin orientation. The magnitude of the ratio $F_{c} / F$, as expected from SPLEED data, will scale with the relative number of spin-polarized electrons of the neighboring atoms, given by the ratio of their magnetic moment $\mu$ (in units of $\mu_{B}$ ) and the atomic number $Z$. The value of $F_{c} / F$ may be estimated from the ratio of the peak heights in the SPEXAFS and EXAFS Fourier transforms, which amounts to $5 \%$ at the $L_{2}$ and $2.5 \%$ at the $L_{3}$ edge. The difference between the $L_{2}$ and $L_{3}$ spectra reflects directly the different photoelectron polarization $P_{e}$ for $\operatorname{Gd} L_{2}$ and $L_{3}$ absorption, ${ }^{3}$ which amounts to $P_{e}\left(L_{2}\right) \sim-0.45$ and $P_{e}\left(L_{3}\right) \sim+0.22$. Taking into account these values one can deduce from the present data a ratio $F_{c} / F$ of about $\sim 0.10$, which is close to the ratio $\mu / Z=0.11$ (in $\mu_{B}$ ) of the $\mathrm{Gd}^{3+}$ ion. From SPLEED data the value of $\lambda_{c} / \lambda$ is expected to be more than 1 order of magnitude smaller and thus can be neglected in our analysis. ${ }^{10}$ A small spin-dependent phase shift $\Phi_{c}$ may be indicated by the small observed difference of $\sim 0.1 \AA$ of the peak positions in EXAFS and SPEXAFS Fourier transforms.

A practical application of the SPEXAFS spectroscopy is shown in Fig. 4 for the case of $\mathrm{Gd}_{3} \mathrm{Fe}_{5} \mathrm{O}_{12} .{ }^{11}$ The Fourier transform of the EXAFS data is dominated by the peak which is due to the eight oxygen neighbors surrounding each Gd atom at an average distance of $2.43 \AA$. The next group of four $\mathrm{Fe}^{3+}$ ions at $3.49 \AA$, four $\mathrm{Fe}^{3+}$ at $3.82 \AA$, and four $\mathrm{Gd}^{3+}$ at $3.82 \AA$ is identified by the smaller peak around $3.08 \AA$. There are an additional two $\mathrm{Fe}^{3+}$ at $3.12 \AA$, which do not contribute significantly to the EXAFS. In the SPEXAFS spectrum strong oscillations and only a small negative background are observed. No significant correlation is found between the periodicity of the $\chi(k)$ and the spin-dependent absorption profiles in the $\mathrm{Gd}_{3} \mathrm{Fe}_{5} \mathrm{O}_{12}$. The Fourier transform of the SPEXAFS contains no significant maximum at the position of the oxygen neighbors, but a dominant peak is found at the distance $r=3.20 \AA$ of the nearest magnetic neighbors. Furthermore, the next group of magnetic neighbors (four $\mathrm{Fe}^{3+}$ ions at $5.62 \AA$, eight $\mathrm{Fe}^{3+}$ at $5.83 \AA$, and eight $\mathrm{Gd}^{+}$at $5.83 \AA$ ) is indicated. These results clearly show that the SPEXAFS probes the local magnetic environment of the absorbing atoms. In the case of a pure ferromagnet such as Gd metal, where the electronic neighbors are identical to the magnetic ones, the spin-dependent absorption profiles contain the same information on the next-neighbor positions as the corresponding normal $\chi(k)$ spectra. However, as demonstrated for the case of $\mathrm{Gd}_{3} \mathrm{Fe}_{5} \mathrm{O}_{12}$, a comparison of the simultaneously measured spin-independent and spindependent absorption profiles allows one to distinguish clearly between magnetic and nonmagnetic neighbors. Thus the SPEXAFS spectroscopy may become a unique tool for the investigation of novel scientifically and technologically interesting magnetic materials.

This work was funded under Contract No. 05 366EA I by the Bundesministerium für Forschung und Technologie, Bonn, Federal Republic of Germany.

${ }^{1}$ G. Schütz, W. Wagner, W. Wilhelm, P. Kienle, R. Zeller, R. Frahm, and G. Materlik, Phys. Rev. Lett. 58, 737 (1987).

${ }^{2}$ G. Schütz, R. Wienke, W. Wilhelm, W. Wagner, P. Kienle, R. Zeller, and R. Frahm, Z. Phys. B (to be published).

${ }^{3}$ G. Schütz, M. Knülle, R. Wienke, W. Wilhelm, W. Wagner, P. Kienle, and R. Frahm, Z. Phys. B 73, 67 (1988).

${ }^{4}$ U. Fano, Phys. Rev. 178, 131 (1969).

${ }^{5}$ S. F. Alvarado, R. Feder, H. Hopster, F. Ciccacci, and H. Pleyer, Z. Phys. B 49, 129 (1982).

${ }^{6}$ J. Unguris, D. T. Pierce, and R. J. Celotta, Phys. Rev. B 29, 1381 (1984).

${ }^{7}$ D. Weller and S. F. Alvarado, Phys. Rev. B 37, 9911 (1988).

${ }^{8}$ R. Frahm, P. Rabe, and R. Haensel, J. Phys. F 14, 1029 (1984).

${ }^{9}$ P. Rabe, G. Tolkiehn, and A. Werner, J. Phys. C 12, 899 (1979).

10J. A. D. Matthew, Phys. Rev. B 25, 3326 (1982).

${ }^{11}$ G. Winkler, Magnetic Garnets (Vieweg \& Sohn, Braunschweig, 1981). 